\title{
OPTIMAL PLACEMENT OF PROFILE MONITORS IN A MISMATCHED FODO LATTICE*
}

\author{
K. Bertsche, Fermi National Accelerator Laboratory ${ }^{\dagger}$, P.O. Box 500, Batavia, IL 60510
}

\section{Abstract}

The beam delivered to a circular machine from a beamline must be matched to the machine lattice function in order to avoid emittance dilution. One method of matching is to rely on beam profile measurements at a number of points in the beamline. In this paper we discuss the expected errors associated with various placement options for beam profile monitors. Examples are given from the Low Energy Booster (LEB) to Medium Energy Booster (MEB) transfer line at the Superconducting Super Collider (SSC).

\section{INTRODUCTION}

Transverse emittance growth arises from three different types of injection mismatch; focusing errors, dispersion errors, and steering errors [1]. For injection into the MEB, it was desired to limit emittance growth from injection mismatches to less than 5\%. Since steering errors could have been detected non-invasively with beam profile monitors (BPMs) and dispersion errors would not have caused very much emittance growth due to the small energy spread in the MEB, focusing errors were the greatest concern. Focusing errors could have been detected with destructive single-turn-only measurements in the MEB using beam profile monitors, or could have been minimized by relying on flying wire measurements and tuning the transfer line with multiple injections. But a less invasive and faster method of detecting errors was desired. It was desired to be able to predict and correct focusing errors on the basis of beam profile measurements in the injection line to the MEB. It was also desired to use these same monitors to measure the emittance of the injected beam. At least three monitors are needed for these measurements, since there are three independent beam parameters $(\alpha, \beta$, and $\varepsilon)$.

The LEB-MEB transfer line was designed as a FODO lattice with $90^{\circ}$ phase advance per cell. A matching section for $\alpha$ and $\beta$ at the upstream end of the transfer line was used to accommodate different possible tunes of the LEB. There was no such matching section at the downstream end. In order for beam to be properly matched into the

* Work performed at the SSC Laboratory, operated by Universities Research Association, Inc. for the U.S. Department of Energy under Contract No. DE-AC3589ER40486.

$\dagger$ Operated by the Universities Research Association, Inc. under contract with the U.S. Department of Energy.
MEB lattice, it was mismatched to the transfer line lattice (i.e. the beam waists were not at the centers of the quadrupoles, and there were large beta waves along the transfer line). Dispersion was matched by tuning pairs of quadrupoles separated by $180^{\circ}$ [2]. In the discussion to follow, it is assumed that the transfer line, downstream of the matching section, has already been tuned to a reference value using a procedure such as that in [3].

It was not obvious a priori where was best to place the profile monitors in the transfer line, in part because beam in this line was designed to be mismatched. A number of philosophies exist for placing profile monitors in a lattice. One is to separate the monitors equally in a $2 \psi$ phase diagram, i.e. to space three monitors by $60^{\circ}$ in phase. A second approach is to place the monitors regularly in the beamline lattice. A third approach is to separate two monitors by $90^{\circ}$ in phase, and to place the third at a separation of $45^{\circ}$ from one of the others. Because of the finite resolution of a profile monitor, different placements of profile monitors will lead to different errors in determination of $\alpha, \beta$, and $\varepsilon$, and errors in $\alpha$ and $\beta$ will lead to emittance growth.

\section{EMITTANCE GROWTH}

An amplitude function mismatch of injected beam into a circular machine (i.e. incorrect $\alpha$ and $\beta$ ) causes an emittance growth. Over time, the beam will filament in phase space and assume the $\beta$ functions of the machine lattice. The beam width at every point in the machine will have grown proportionally by an amount [1; Eq. 7.56]:

$$
\frac{\sigma^{2}}{\sigma_{0}^{2}}=1+\frac{1}{2}|\operatorname{det} \Delta J|
$$

where $\sigma_{0}$ is the initial standard deviation of the beam width, $\sigma$ is the standard deviation including effects of mismatch, and $\Delta J$ is the error matrix:

$$
\Delta J=\left(\begin{array}{cc}
\Delta \alpha & \Delta \beta \\
-\Delta \gamma & -\Delta \alpha
\end{array}\right)
$$

Using the relations:

$$
\beta \gamma=1+\alpha^{2}
$$

and

$$
\sigma^{2}=\sigma_{0}^{2} \frac{\varepsilon}{\varepsilon_{0}}=\sigma_{0}^{2}(1+f)
$$

where $f$ is the fractional emittance growth, Eq. (1) may be rewritten to give the emittance growth resulting from this mismatch: 


$$
\begin{aligned}
f & =\frac{1}{2}\left|\Delta \alpha^{2}-2 \frac{\alpha}{\beta} \Delta \alpha \Delta \beta+\frac{\left(1+\alpha^{2}\right)}{\beta^{2}} \Delta \beta^{2}\right| \\
& =\frac{1}{2}\left|\frac{\Delta \beta^{2}+(\beta \Delta \alpha-\alpha \Delta \beta)^{2}}{\beta^{2}}\right|
\end{aligned}
$$

While Eq. (5) is applicable at any point in the circular machine lattice, it will be convenient for later calculations if it is applied at the center of a quadrupole (e.g. the first quadrupole in the circular machine), where (nominally) $\alpha=$ 0 . This eliminates troublesome cross-terms involving errors in both $\alpha$ and $\beta$ and Eq. (5) becomes:

$$
f=\frac{1}{2}\left|\Delta \alpha^{2}+\frac{\Delta \beta^{2}}{\beta^{2}}\right|
$$

which can also be interpreted as a relation between the mean emittance growth and the rms errors in $\alpha$ and $\beta$ of the injected beam, measured at the center of the first quadrupole in the circular machine.

\section{BEAM PROPAGATION}

For a beam confined in a periodic lattice, the optical transformation between two different longitudinal locations 1 and 2 may be written in terms of the Courant-Snyder parameters $\alpha, \beta$, and $\psi$ at the two locations [4]:

$$
\left.\begin{array}{l}
M_{1 \rightarrow 2}= \\
\sqrt{\frac{\beta_{2}}{\beta_{1}}}\left(\begin{array}{c}
\cos \Delta \psi+ \\
\alpha_{1} \sin \Delta \psi
\end{array}\right) \\
\begin{array}{c}
\left(1+\alpha_{1} \alpha_{2}\right) \sin \Delta \psi \\
+\left(\alpha_{2}-\alpha_{1}\right) \cos \Delta \psi \\
-\frac{\sqrt{\beta_{1} \beta_{2}}}{\sqrt{\beta_{1} \beta_{2}}} \sin \Delta \psi
\end{array} \\
\frac{\beta_{1}}{\beta_{2}}\left(\begin{array}{c}
\cos \Delta \psi- \\
\alpha_{2} \sin \Delta \psi
\end{array}\right)
\end{array}\right)
$$

This transformation may be calculated in terms of the actual beam parameters $(\alpha, \beta, \psi)$ or in terms of the reference parameters $(\alpha, \beta, \psi)$ for a perfectly-tuned beam.

The phase space ellipse of a beam may be defined by a matrix $E$ which may be expressed as [4]:

$$
E=\left(\begin{array}{ll}
\sigma_{x}^{2} & \sigma_{x x^{\prime}} \\
\sigma_{x x^{\prime}} & \sigma_{x}^{2}
\end{array}\right)=\left(\begin{array}{ll}
\sigma_{11} & \sigma_{12} \\
\sigma_{21} & \sigma_{22}
\end{array}\right)=\frac{\varepsilon}{\pi}\left(\begin{array}{c}
\beta-\alpha \\
-\alpha \gamma
\end{array}\right)
$$

where $\varepsilon$ is the un-normalized rms emittance, $\sigma_{x}$ is the rms beam width in the absence of dispersion, and $\alpha, \beta, \gamma$ are the ellipse coefficients, normalized as in Eq. 3.

The beam matrix $E$ evolves from location 1 to location 2 according to the expression:

$$
E_{2}=M_{1 \rightarrow 2} E_{1} M_{1 \rightarrow 2}^{t}
$$

Transforming matrix $E_{1}$ to $E_{2}$, using Eqs. (7) and (9), and examining the $\sigma_{11}$ term of $E_{2}$, one finds that a measurement of the beam width at location 2 gives, in terms of the beam parameters at location 1 :

$$
\sigma_{x \mid}^{2}=\sigma_{x \mid 1}^{2} m_{11}^{2}+2 \sigma_{x x \mid 1} m_{11} m_{12}+\sigma_{x \mid 1}^{2} m_{12}^{2}
$$

or, substituting from Eqs. (7) and (8),

$$
\begin{aligned}
\sigma_{x \mid}^{2} & =\frac{\varepsilon_{b} \beta_{b \mid 1}}{\pi} \frac{\beta_{2}}{\beta_{1}}\left(\cos \Delta \psi+\alpha_{1} \sin \Delta \psi\right)^{2} \\
& -\frac{2 \varepsilon_{b} \alpha_{b \mid 1}}{\pi} \beta_{2} \sin \Delta \psi\left(\cos \Delta \psi+\alpha_{1} \sin \Delta \psi\right) \\
& +\frac{\varepsilon_{b}\left(1+\alpha_{b}^{2}\right)}{\pi \beta_{b \mid 1}} \beta_{1} \beta_{2} \sin ^{2} \Delta \psi
\end{aligned}
$$

Note that the parameters $(\alpha, \beta, \psi)$ of the beamline (i.e. the parameters for a perfectly-tuned beam which exhibits no emittance growth) have been kept distinct from those of the possibly-mistuned beam $\left(\alpha_{b}, \beta_{b}, \varepsilon_{b}\right)$ measured at location 1. We assume that the beamline has been previously tuned very accurately using a procedure similar to that in [3], so that beamline parameters at all points are known to high precision.

\section{MEASUREMENT ERRORS}

Eq. (11) may be expanded to first order in the beam parameters $\left(\alpha_{b}, \beta_{b}, \varepsilon_{b}\right)$, giving a relation between beam width errors at a point 2 and errors in $\left(\alpha_{b}, \beta_{b}, \varepsilon_{b}\right)$ at a remote location 1 . Location 1 may be taken to be the center of the first quadrupole in the circular machine, where $\alpha=0$, and location 2 may be taken to be a general point upstream of this in the injection line, giving:

$$
\begin{aligned}
\frac{\Delta \sigma_{x R}}{\sigma_{x k}}= & \Delta \alpha_{b \mid 1}(\sin \Delta \psi \cos \Delta \psi) \\
& +\frac{\Delta \beta_{b \mid 1}}{\beta_{b \mid 1}}\left(\frac{\cos ^{2} \Delta \psi-\sin ^{2} \Delta \psi}{2}\right)+\frac{1}{2} \frac{\Delta \varepsilon_{b}}{\varepsilon_{b}} \\
= & \Delta \alpha_{b \mid 1}\left(\frac{\sin (2 \Delta \psi)}{2}\right) \\
& +\frac{\Delta \beta_{b \mid 1}}{\beta_{b \mid 1}}\left(\frac{\cos (2 \Delta \psi)}{2}\right)+\frac{1}{2} \frac{\Delta \varepsilon_{b}}{\varepsilon_{b}}
\end{aligned}
$$

where it has been assumed that the beam parameters at location 1 are nominally those of the reference tune (i.e. tuning errors are assumed to be small).

A similar expression may be written for each of the three profile monitors assumed to be in the transfer line at locations A, B, and C, giving the errors in beam widths at each of the monitors corresponding to injection errors into the circular machine. This may be written as a matrix:

$$
\left(\begin{array}{c}
\Delta \sigma_{x \mid A} / \sigma_{x \mid A} \\
\Delta \sigma_{x \mid B} / \sigma_{x \mid B} \\
\Delta \sigma_{x \mid C} / \sigma_{x \mid C}
\end{array}\right)=M\left(\begin{array}{c}
\Delta \alpha_{b \mid 1} \\
\Delta \beta_{b \mid 1} / \beta_{b \mid 1} \\
\Delta \varepsilon_{b} / \varepsilon_{b}
\end{array}\right)
$$

In practice, one measures the beam widths and wishes to calculate the resultant errors in focusing parameters. Thus the above matrix needs to be inverted: 


$$
\left(\begin{array}{c}
\Delta \alpha_{b \mid 1} \\
\Delta \beta_{b \mid 1} / \beta_{b \mid 1} \\
\Delta \varepsilon_{b} / \varepsilon_{b}
\end{array}\right)=M^{-1}\left(\begin{array}{c}
\Delta \sigma_{x \mid A} / \sigma_{x \mid A} \\
\Delta \sigma_{x \mid B} / \sigma_{x \mid B} \\
\Delta \sigma_{x \mid C} / \sigma_{x \mid C}
\end{array}\right)
$$

This equation may be used to find errors in $\alpha, \beta$, or $\varepsilon$ based on measured deviations in $\sigma$. Alternatively, terms may be added in quadrature to give the measurement tolerance of $\alpha, \beta$, and $\varepsilon$ based on the rms measurement precision of $\sigma$, and $\mathrm{rms} \alpha$ and $\beta$ tolerances may be combined by use of Eq. (6) to give the resultant mean emittance growth. The placement of profile monitors is optimum when emittance growth and/or emittance measurement tolerance are minimized.

For the case where the fractional error in $\sigma$ is constant, Eq. (14) depends only on the phase advances, and it can be shown that spacing the profile monitors by $60^{\circ}$ is optimal for minimizing both emittance measurement tolerance and emittance growth. But for many situations, including the MEB, this condition is not met. It was believed that measurements with MEB profile monitors would give smaller fractional errors for larger $\sigma$, (a fixed absolute error of $0.1 \mathrm{~mm}$ was assumed below, though in general an error proportional to $\sigma^{1 / 2}$ would be more realistic [5]).

Since the fractional errors in $\sigma$ depend on $\beta$, Eq. (14) depends on $\beta$ as well as on phase advance. The equations are highly lattice-dependent, and it is impossible to find a general solution. The matrices in Eqs. (13) and (14) were evaluated and inverted numerically for various placements of profile monitors based on the nominal lattice functions of the LEB-MEB transfer line [2]. The results are shown in Tables 1 and 2 . In these tables, focusing ( $\alpha$ and $\beta$ ) errors have been converted to mean emittance growth on the basis of Eq. (6). Shown are the mean emittance growth upon injection to the MEB and the rms error in emittance measurement, resulting from an assumed measurement precision of $0.1 \mathrm{~mm} \mathrm{rms} \mathrm{in} \sigma$ at each profile monitor.

\section{Profile Monitor Placement-Horizontal Plane}

\begin{tabular}{|l|c|c|}
\hline Placement & $\varepsilon$ growth & $\varepsilon$ msmt \\
\hline Adjacent to quadrupoles & $23 \%$ & $57 \%$ \\
\hline$\sim 60^{\circ}$ phase separation & $14 \%$ & $27 \%$ \\
\hline$\sim 45^{\circ}$ and $90^{\circ}$ separation & $9 \%$ & $30 \%$ \\
\hline Best location found & $8 \%$ & $30 \%$ \\
\hline
\end{tabular}

Table 1: Comparison of various placements of three horizontal profile monitors in LEB-MEB transfer line.

For the horizontal plane in the LEB-MEB transfer line, placing three beam profile monitors with phase separations of about $45^{\circ}$ and $90^{\circ}$ gave good measurements, but this arrangement was able to be improved upon slightly by trial and error adjustment of the profile monitor locations.

\section{Profile Monitor Placement-Vertical Plane}

\begin{tabular}{|l|c|c|}
\hline Placement & $\varepsilon$ growth & $\varepsilon$ msmt \\
\hline Adjacent to quadrupoles & $6 \%$ & $20 \%$ \\
\hline$\sim 60^{\circ}$ phase separation & $7 \%$ & $19 \%$ \\
\hline$\sim 45^{\circ}$ and $90^{\circ}$ separation & $8 \%$ & $22 \%$ \\
\hline Best location found & $6 \%$ & $20 \%$ \\
\hline
\end{tabular}

Table 2: Comparison of various placements of three vertical profile monitors in LEB-MEB transfer line.

For the vertical plane, where the reference beam had a different pattern of beta waves in the transfer line, regular lattice locations adjacent to three quadrupoles gave better measurements. Other arrangements of three profile monitors were found which gave roughly the same sensitivity to errors, but no arrangements were found which were better.

In neither plane can $\alpha$ and $\beta$ be measured to sufficient precision, on the basis of a single measurement, to limit emittance growth to 5\%. However, assuming that the errors in measurement of $\sigma$ at each profile monitor were random, a small number of measurements could have been averaged to statistically reduce these errors to less than $5 \%$.

\section{CONCLUSIONS}

If measurements of beam $\sigma$ have constant fractional errors, profile monitors should be placed equally in $2 \psi$ to give the best detection sensitivity for focusing errors; i.e. three monitors should each be separated by $60^{\circ}$ in phase. For measurements which do not have constant fractional errors, the best placement depends on the details of the lattice functions. Different placements of profile monitors may be evaluated numerically following the procedure outlined here to find the best arrangement.

\section{REFERENCES}

[1] D.A. Edwards and M.J. Syphers, An Introduction to the Physics of High Energy Accelerators, Wiley, 1993.

[2] N. Mao et al, "Beam Optics of LEB-MEB Transfer Line for Superconducting Super Collider", Proceedings of the 1993 Particle Accelerator Conference, Washington, D.C., vol. 1, pp. 333-335.

[3] K. Bertsche and N. Mao, "Accurate Tuning of $90^{\circ}$ Cells in a FODO Lattice", these proceedings.

[4] K.L. Brown and R. Servranckx, 1990 US Particle Accelerator School class notes.

[5] K. Bertsche and J. Palkovic, "Beam Profile Measurement in the Presence of Noise", these proceedings. 